\title{
Sobre el progreso en las ciencias fisiológicas*
}

El método experimental, que desde hace mucho tiempo se aplica con tanto éxito en el estudio de los fenómenos de los cuerpos brutos tiende hoy, cada vez más, a introducirse en el estudio de los fenómenos de los seres vivos. Pero muchos científicos dudan aún de su utilidad real porque piensan que la espontaneidad vital será siempre un obstáculo insuperable para la aplicación de un método de investigación que sea común a las ciencias fisiológicas y a las ciencias físico-químicas. Debido a que los cuerpos brutos están desprovistos de espontaneidad, las manifestaciones de sus propiedades permanecen encadenadas, de una manera absoluta, a las variaciones de las circunstancias del medio que los rodea, lo que permite al experimentador acceder a ellos fácilmente, modificándolos a voluntad. Los seres vivos, por el contrario, dotados de espontaneidad, parecen estar provistos de una fuerza interior que hace que las manifestaciones de vida sean más independientes de las variaciones de las influencias exteriores cuanto más se eleva el ser vivo en la escala de organización. Tanto en el hombre como en los animales superiores, esta fuerza vital parece tener como resultado que el cuerpo vivo escape a las influencias físico-químicas generales, tornándose así inaccesible a los procedimientos ordinarios de experimentación.

Por otra parte, todos los fenómenos de los animales vivos están conectados por la sensibilidad y mantenidos por ella en una armonía reciproca tal que parece imposible separar una parte del organismo sin provocar una perturbación en todo su conjunto. Y muchos médicos y naturalistas explotaron estos argumentos para oponerse al empleo de la experimentación en los seres vivos. Consideraron que la fuerza vital estaba en oposición con las fuerzas físico-químicas, que ella dominaba todos los fenómenos de la vida, que la sometía a leyes especiales, y eso hacía del organismo un todo viviente al cual el experimentador no podía tocar sin destruir a la propia vida. Cuvier, que compartió esta opinión, y que pensaba que la fisiología debía ser una ciencia de observación y de deducción anatómica, se expresa de la siguiente manera: "Todas las partes de un cuerpo vivo están conectadas; ellas sólo pueden actuar conjuntamente. Querer aislar una de ellas, separándola de esa totalidad, es colocarla en el orden de las sustancias muertas, es cambiar totalmente su esencia". ${ }^{1}$ Y, si esas objeciones precedentes fuesen fundadas, habría que reconocer que, o bien no existe ningún determinismo posible en los fenómenos de la vida, lo que sería negar pura y simplemente la fisiología experimental; o bien habría que admitir que la fuerza vital debe ser estudiada según un método particular y que la ciencia de los cuerpos vivos debe basarse en principios distintos a los de la ciencia de los cuerpos inertes.

Estas ideas, que fueron florecientes en otras épocas, hoy, bajo la influencia del progreso fisiológico, se desvanecen progresivamente. Sin embargo, es importante extirpar sus últimos gérmenes, porque lo que queda aún de esas ideas, en ciertos espíritus, constituye un verdadero obstáculo para la marcha de la ciencia fisiológica y la medicina experimental. Por eso me propongo demostrar que los fenómenos de los cuerpos

\footnotetext{
* Traducción de "Du progrès dans les sciences physiologiques", primeramente publicado en la Revue des deux mondes (58(35)(1865): 640-663), y luego reeditado en La science expérimental (Bernard, C., La science expérimental, Paris: Baillière, pp.38-98). [N. de T.]

${ }^{1}$ Carta de Cuvier a J. C. Mertrud, Lecciones de anatomía comparada, p. 5, París, año VIII. [El año mencionado corresponde al calendario republicano francés. Se sabe que la carta fue escrita el "28 de ventoso del año VIII", o sea, el 19 de marzo del año 1800 en el calendario gregoriano. N. de T.]

Metatheoria 11(2)(2021): 35-49. ISSN 1853-2322. eISSN 1853-2330.

(C) Editorial de la Universidad Nacional de Tres de Febrero.

(C) Editorial de la Universidad Nacional de Quilmes.

Publicado en la República Argentina.
} 
vivos, tal como los fenómenos de los cuerpos brutos, están sometidos a un determinismo absoluto y necesario. La ciencia vital no puede emplear otros métodos, ni tener otras bases, que no sea las de la propia ciencia mineral; y no hay que establecer ninguna diferencia entre los principios de las ciencias fisiológicas y los de las ciencias físico-químicas.

La espontaneidad de los seres vivos no debe impedir que el fisiólogo aplique del método experimental. ${ }^{2}$ Efectivamente, pese a esa espontaneidad, los seres vivos no son independientes de las influencias del mundo exterior; y sus funciones están constantemente relacionadas con las condiciones que reglan su aparición de una manera determinada y necesaria. A partir del momento en que entramos en el estudio de los mecanismos propios a los fenómenos de la vida, percibimos que la espontaneidad aparente de la que disfrutan los cuerpos vivos no es más que la consecuencia natural de ciertas circunstancias bien determinadas; y nos será fácil probar que, en el fondo, las manifestaciones de los cuerpos vivos, tanto como las manifestaciones de los cuerpos brutos, están ligadas a condiciones de orden puramente físico-quimico. Y veremos, además, el problema que se plantean el fisiólogo y el médico experimentador, no es el de llegar hasta la causa primera de la vida, sino el de conocer las condiciones físico-químicas determinantes de la actividad vital.

Notemos en primer lugar que la independencia del ser vivo en el medio ambiente cósmico aparece únicamente en los organismos completos y superiores. En los seres inferiores, reducidos a un organismo elemental, tales como los infusorios, no hay una independencia real. Estos seres no siempre manifiestan esas propiedades vitales, a menudo muy activas, de las que ellos sólo llegan a estar dotados sólo bajo la influencia de la humedad, la luz, el calor exterior; siendo que, si una de estas condiciones falta, la manifestación vital cesa porque los fenómenos físico-químicos, de los que dicha manifestación depende, se detienen. Entonces, muchos de estos animales caen en un estado de vida latente que no es otra cosa que un estado de indiferencia química del cuerpo organizado frente al mundo exterior. Esta suspensión completa de las manifestaciones de la vida puede durar un tiempo en cierta forma indefinido. Bajo la influencia de solamente una gota de agua, Spallanzani vio reaparecer la vitalidad en los gusanos del trigo que habían permanecidos inertes y desecados desde hacía casi treinta años. ${ }^{3}$ El agua restituida a esos cuerpos, hizo reaparecer los fenómenos químicos, permitiendo que los tejidos manifestasen sus propiedades vitales.

En los vegetales, las manifestaciones de los fenómenos de la vida también están ligadas a las condiciones de calor, de humedad y de luz del medio ambiente; y ahí radica esa influencia de las estaciones que todo el mundo conoce. Sucediendo lo mismo, además, con los animales de sangre fría: en ellos, los fenómenos de la vida se adormecen o se activan según las condiciones climáticas de calor, frio, humedad o sequedad. El agua, el calor, y la electricidad, que son también los excitantes de los fenómenos físico-químicos, influencian, acelerando o desacelerando las manifestaciones vitales de los seres vivos; y lo hacen del mismo modo en que provocan, acelerando y desacelerando, las manifestaciones minerales en los cuerpos brutos. En lugar de ver, como los vitalistas, una especie de oposición o de incompatibilidad entre las condiciones de las funciones vitales y las condiciones de las acciones minerales, hay que admitir que, entre esos dos órdenes de fenómenos, hay un paralelismo completo y una relación directa y necesaria.

\footnotetext{
2 Para la demostración técnica de estas consideraciones, remito al lector a mi obra: Introducción al estudio de la medicina experimental, París, 1865.

3 Spallanzani, Observaciones y experimentos sobre algunos animales sorprendentes que el observador puede, a su voluntad, pasarlo de la muerte a la vida. Obras, in- $8^{\circ}$, p. 203.
}

Metatheoria 11(2)(2021) 
Esta relación es más estrecha entre los seres inferiores, entre los vegetales y entre los animales de sangre fría. Pero, en el hombre y en los otros animales de sangre caliente, hay, en general, una independencia evidente entre las funciones del organismo y las condiciones del medio ambiente. En estos casos, los fenómenos vitales no sufren la influencia del cambio de las estaciones o de las variaciones cósmicas. En virtud de un mecanismo protector más completo, el animal posee y mantiene, en un medio interior que le es propio, las condiciones de humedad y de calor necesarias para las manifestaciones de los fenómenos vitales. El organismo del animal de sangre caliente, estando lo suficientemente protegido, difícilmente entra en equilibrio con el medio exterior; sino que él mantiene sus órganos en una suerte de invernadero, y de esta manera protege sus actividades vitales. Y eso es semejante a lo que ocurre en los invernaderos de nuestros jardines; donde vemos manifestarse una actividad vegetativa, que es independiente de los calores y las heladas del exterior, y que está ligada, de manera íntima y necesaria, a las condiciones físico-químicas de la atmosfera interior de ese ambiente artificial.

Las manifestaciones de la vida que observamos en el hombre, o en el animal superior, son mucho más complejas de lo que nos parecen; pero jamás hay que olvidar que, cualquiera sea su complejidad, dichas manifestaciones siempre son el resultado de las propiedades intimas de una multitud de elementos orgánicos cuya actividad está ligada a las condiciones físico-químicas de los medios internos en los que dichos elementos están sumergidos. Por eso, si suprimimos de nuestras explicaciones ese medio interior que no vemos, considerando sólo el medio exterior que está bajo nuestros ojos, podemos ser conducidos al error de creer que en el ser vivo hay una fuerza vital que viola las leyes físico-químicas del medio cósmico general.

Las máquinas vivientes están creadas y construidas de tal manera que, perfeccionándose, se vuelven cada vez más libres en el mundo exterior. Pero, esa determinación vital del medio interno sólo existe en virtud de ese perfeccionamiento que progresivamente lo fue aislando del medio cósmico general. Las maquinas que la inteligencia del hombre crea, aunque son mucho más toscas, también poseen una independencia resultante del funcionamiento de su mecanismo interior. Una máquina de vapor posee una actividad independiente de las condiciones físico-químicas del medio exterior: haga frío o calor, esté seco o húmedo, la máquina sigue andando. Pero, para el físico que desciende al interior de la máquina, esta independencia es sólo aparente; y el movimiento de cada engranaje interior está determinado por condiciones físicas absolutas cuyas leyes él conoce. Y lo mismo ocurre con el fisiólogo. Si éste pudiese introducirse en el medio interior de la maquina viva, encontraría un determinismo que para él sería la base real de la ciencia experimental de los cuerpos vivos.

Para comprender la experimentación en los seres vivos de organización superior, es necesario tener en cuenta dos medios: el medio cósmico o extra-orgánico, que es común a los seres vivos y a los seres brutos; y el medio intra-orgánico, que es privativo de los seres vivos. Este último medio se relaciona con los elementos orgánicos activos: músculos, nervios, glándulas, etc., y está formado por todos los líquidos intra-orgánicos y blastemáticos. ${ }^{4}$ Encontramos en ese medio liquido las condiciones de temperatura, el aire y los alimentos disueltos en el agua; porque, como ya dijimos en otra oportunidad, ${ }^{5}$ todos los elementos orgánicos activos que componen nuestro organismo son necesariamente acuáticos, y es únicamente por un artificio de construcción que nuestro cuerpo puede existir y moverse en el aire seco. Por eso, la medicina experimental, o científica, deberá siempre basarse en el conocimiento de las propiedades del medio intra-orgánico.

Cuando un medicamento ejerce su acción sobre nosotros, él no actúa sobre nuestro estómago, sino sobre nuestro medio intra-orgánico. Lo hace después de haber penetrado en nuestra sangre y de haberse puesto

\footnotetext{
${ }^{4}$ Ver Claude Bernard, Lecciones de fisiología experimental aplicada a la medicina, París, 1855-1856, 2 vols.; Lecciones sobre la fisiología y la patología del sistema nervioso, París, 1858, 2 vols.; Lecciones sobre las propiedades fisiológicas de los líquidos del organismo, París, 1859, 2 vols.

${ }^{5}$ Ver Estudio sobre la fisiología del corazón, p. 316.
} 
en contacto con nuestras partículas organizadas. Esta idea del medio interior, que ha guiado mis estudios en fisiología, me sirvió para determinar, de una manera más precisa, la acción de las sustancias toxicas sobre los diversos elementos de nuestro cuerpo. ${ }^{6}$ Pero de ahí también surgieron otras nuevas consideraciones que están destinadas a guiar al fisiólogo en sus experimentos y a servir de base, al mismo tiempo, a la fisiología y la patología generales. En efecto, desde el punto de vista médico o terapéutico, no cabe encontrar, ni en el hombre, ni en los animales superiores, una independencia vital en relación con los venenos y los medicamentos. Todos los días podemos modificar o anular los fenómenos de la vida, haciendo penetrar substancias activas en nuestra sangre o en nuestro medio orgánico. Pero sería una ilusión si, en esas modificaciones tan variadas y múltiples del organismo, viésemos la expresión indeterminada de una fuerza vital cualquiera. ${ }^{7}$ Todas esas modificaciones dependen de las condiciones físico-químicas precisas que tienen lugar en nuestro medio interior o en los elementos histológicos de nuestros tejidos.

En el pasado, Buffon había creído que debía existir en el cuerpo de los seres vivos un elemento orgánico particular que no se encontraría en los cuerpos minerales. ${ }^{8}$ Los progresos de las ciencias químicas destruyeron esta hipótesis mostrando que el cuerpo vivo está constituido, exclusivamente, por materias simples o elementales extraídas del mundo mineral. Y también se llegó a creer en la actividad de una fuerza especial para las manifestaciones de los fenómenos de la vida; pero el progreso de las ciencias fisiológicas destruyó también esta segunda hipótesis, haciéndonos ver que las propiedades vitales no tienen ninguna espontaneidad por ellas mismas, sino que la tienen en virtud de propiedades minerales. Siendo esas mismas condiciones físico-químicas generales las que presiden las manifestaciones de ambos tipos de propiedades. Sin embargo, de eso que acabamos de decir no se podría inferir que nosotros asimilamos los cuerpos vivos a los cuerpos brutos; el buen sentido de todos reaccionaria inmediatamente ante semejante confusión. Es evidente que los cuerpos vivos no se comportan como los cuerpos inanimados. Se trata solamente de caracterizar y de definir sus diferencias, ya que ese es el punto capital para comprender cabalmente a la fisiología experimental.

De todas las definiciones de la vida, la que es menos comprometedora, y la más verdadera, es la que dio la Enciclopedia: "la vida es lo contrario a la muerte". Esta definición es de una claridad inocente; y sin embargo no podríamos decir nada mejor, porque nunca sabremos lo que es la vida en sí misma. Para nosotros un cuerpo está vivo porque puede morir y porque está organizado de tal manera que, por el juego natural de sus funciones, preserva su organización por un tiempo y se perpetua con la formación de individuos que se le parecen. La vida tiene entonces su esencia en la fuerza, o más bien en la idea directriz del desarrollo orgánico; es la fuerza vital así comprendida que constituía la vis medicatrix de Hipócrates, la fuerza seminal y el archeus faber de Van Helmont. Por eso, si yo tuviera que definir la vida en una sola palabra diría: la vida es la creación. En efecto, la vida para el fisiólogo no sería otra cosa que la causa primera creadora del organismo que se nos escapa siempre, tal como ocurre con todas las causas primeras. Esa causa se manifiesta en la organización: mientras perdura, el ser vivo está bajo el imperio de esta influencia vital creadora; y la muerte natural llega cuando la creación orgánica ya no puede realizarse.

El espíritu del hombre no puede concebir un efecto sin causa: la visión de un fenómeno despierta siempre en él la idea de causalidad; y toda la ciencia humana consiste en remitir los efectos observados a sus causas. Pero desde siempre los filósofos y los científicos han distinguido dos órdenes de causas: las causas primeras y las causas secundarias o próximas. Las causas primeras, que son relativas al origen de las cosas, nos resultan absolutamente impenetrables; y las causas próximas, que se relacionan con las condiciones de

${ }^{6}$ Ver Estudios fisiológicos sobre algunos venenos americanos, el curare, p. 237.

${ }^{7}$ Claude Bernard, Lecciones sobre los efectos de las sustancias toxicas y medicamentosas, París, 1857.

${ }^{8}$ Buffon, Obras completas, publicadas por Lacépède, t. IX, p. 25.

Metatheoria 11(2)(2021) 
manifestación de los fenómenos, están a nuestro alcance y podemos conocerlas experimentalmente. Newton dijo que aquel que se dedique a la investigación de las causas primeras nos muestra, ya sólo por eso, que no es un científico. Efectivamente, esta investigación es estéril porque nos presenta problemas inabordables para el método experimental.

Resumiendo: tanto en el fenómeno vital como en cualquier otro tipo de fenómeno natural, hay dos órdenes de causas. En primer lugar, hay una causa primera creadora, legisladora y rectora de la vida; y que es inaccesible a nuestros conocimientos. Luego, hay una causa próxima, o ejecutora del fenómeno vital; que siempre es de naturaleza físico-química, y que está al alcance del experimentador. La causa primera de la vida nos da la evolución o la creación de la máquina organizada; pero, una vez creada, la máquina funciona en virtud de las propiedades de los elementos que la constituyen y bajo la influencia de las condiciones físico-químicas que actúan sobre esos elementos. Para el fisiólogo y el médico experimentador, el organismo viviente no es más que una máquina admirable que está dotada de las propiedades más maravillosas y que es puesta en marcha con la ayuda de los mecanismos más complejos y más delicados. Es una máquina cuyo mecanismo, ellos deben analizar y determinar, para así poder modificarlo. La muerte accidental es la dislocación, o la destrucción, del organismo debido a la ruptura, o la interrupción, de la acción de uno o varios de sus mecanismos vitales.

\section{II}

La investigación de las causas primeras, ya lo dijimos, no es del dominio científico. Cuando el experimentador alcanza el determinismo de los fenómenos, él no puede ir más allá; y, desde ese punto de vista, el límite de su conocimiento es el mismo tanto en las ciencias de los cuerpos vivos como en las ciencias de los cuerpos brutos. La naturaleza de nuestro espíritu nos lleva, en primer lugar, a investigar la causa primera, es decir la esencia y el porqué de las cosas. De ese modo, apuntamos más allá del objetivo que nos es dado alcanzar; y rápidamente la experiencia nos enseña que no podemos ir más allá del cómo; es decir: no podemos ir más allá del determinismo que nos da la causa próxima, o la condición de la existencia de los fenómenos. Lo que nosotros llamamos determinismo de un fenómeno no es otra cosa que la causa determinante o la causa próxima; es decir: la circunstancia que determina la aparición de un fenómeno y constituye su condición, o una de sus condiciones, de existencia. La palabra determinismo tiene un significado distinto al de la palabra fatalismo. El fatalismo supone la manifestación necesaria de un fenómeno independiente de sus condiciones, mientras que el determinismo es la condición necesaria de un fenómeno cuya manifestación no es forzosa. El fatalismo es tan anticientífico como el indeterminismo.

Cuando, por un análisis experimental continuado encontramos la causa próxima, o la condición elemental de un fenómeno, hemos alcanzado una meta científica que no podremos superar jamás. Cuando sabemos que el agua, con todas sus propiedades, resulta de la combinación del oxígeno con el hidrógeno en ciertas proporciones, y conocemos la condición de esta combinación, ya sabemos todo lo que podemos saber científicamente sobre este tema. Pero eso responde al cómo y no al porqué de las cosas: sabemos cómo se puede hacer el agua; pero por qué la combinación de un volumen de oxígeno y dos volúmenes de hidrógeno forman agua, eso no lo sabemos: no podemos saberlo y no debemos investigarlo. En medicina, tanto como en química, no es científico preguntarse por qué; ese cuestionamiento no hace más que perdernos en problemas irresolubles y sin aplicación.

Quizá fue para burlarse de esta tendencia anticientífica de la medicina, que resulta de la ausencia del sentimiento de ese límite de nuestros conocimientos, que Molière puso en la boca de su candidato a doctor, 
a quien le preguntaban por qué el opio hacía dormir, la siguiente respuesta: porque hay en él una fuerza dormitiva cuya naturaleza consiste en adormecer los sentidos. Y esa respuesta, que parece divertida o absurda, es, sin embargo, la única que se podría haber dado. De igual manera si quisiésemos responder a esta pregunta: “PPor qué el hidrógeno, combinándose con el oxígeno forma el agua?”, nos veríamos obligados a responder: "Porque hay en el hidrógeno una propiedad capaz de engendrar agua". Lo absurdo es preguntar por qué, puesto que eso entraña una respuesta que parecerá inocente o ridícula. Es mejor reconocer que no lo sabemos y que allí se encuentra el límite de nuestros conocimientos. Podemos saber cómo y en qué condiciones el opio hace dormir, pero nunca sabremos por qué.

Las propiedades de la materia viva no pueden manifestarse ni conocerse sino es a través de sus relaciones con las propiedades de la materia bruta, de donde resulta que las ciencias fisiológicas experimentales tienen como base necesaria a las ciencias físico-químicas de las que toman sus procedimientos de investigación y sus medios de acción. El cuerpo vivo está dotado de propiedades y de facultades propias de su naturaleza, tales como la plasticidad orgánica, la contractibilidad, la sensibilidad, la inteligencia; pero, todas estas propiedades, todas estas facultades, sin excepción, sean del orden que sean, encuentran su determinismo, es decir sus medios de manifestarse y de accionar, en las condiciones físico-químicas del medio exterior e interior del organismo. Pero, no obstante eso, tanto en los fenómenos vitales como en los fenómenos minerales, la condición de existencia del fenómeno no nos enseña nada sobre su naturaleza. Cuando sabemos que la excitación exterior de ciertos nervios y que el contacto físico y químico de la sangre, a cierta temperatura, con los elementos nerviosos cerebrales, son condiciones necesarias para la manifestación del pensamiento y de los fenómenos nerviosos e intelectuales, este echo nos indica el determinismo, o las condiciones de estos fenómenos. Pero eso no nos enseña nada sobre la naturaleza primera de la inteligencia. De igual manera, sabemos que el frotamiento y las acciones químicas producen la electricidad, y eso nos indica el determinismo, o las condiciones del fenómeno, sin nada decirnos sobre la naturaleza primera de la electricidad.

El experimentador puede modificar los fenómenos de la naturaleza que están a su alcance. Pero, por una disposición que sin duda debemos considerar muy sabia, él nunca podrá actuar sobre los cuerpos celestes; y es por eso que la astronomía está condenada a ser una ciencia de observación pura para siempre. "En la tierra, dice Laplace, podemos hacer variar los fenómenos con los experimentos; en el cielo, observamos prolijamente todo lo que nos ofrecen los movimientos celestes". ${ }^{9}$ Entre las ciencias de los fenómenos terrestres a las que podemos considerar ciencias de experimentación, las ciencias minerales fueron las primeras debido a esa simpleza de sus fenómenos, que los hacía accesibles al experimentador. Pero fue un error haber querido excluir la experimentación de la ciencia de los seres vivos, diciendo que el organismo se aísla como un pequeño mundo, un microcosmos, dentro del macrocosmos, y que su vida representa el resultado de un todo, o de un sistema indivisible, del que únicamente podemos observar los efectos sin modificarlos.

Si la medicina, por ejemplo, quisiese seguir siendo una ciencia de observación, los médicos deberían contentarse con observar a sus enfermos, limitándose a predecir la marcha y el fin de sus enfermedades, sin tocar nada ahí, tal como el astrónomo no toca a sus planetas. Cuando el médico experimental da un remedio activo, él realiza una verdadera experimento tratando de modificar alguno de los síntomas de la enfermedad. La experimentación científica debe estar fundada en el conocimiento del determinismo de los fenómenos, de lo contrario la experimentación seria ciega y empírica. El empirismo debe ser admitido como un período necesario de la evolución de la medicina experimental, pero no debería ser erigido en sistema, como lo quisieron algunos médicos. La experimentación puede ser aplicada a todos los fenómenos naturales de

\footnotetext{
9 Laplace, Sistema del mundo, cap. II
}

Metatheoria 11(2)(2021) 
cualquier tipo que sean; y eso es así porque el experimentador no engendra los fenómenos: él actúa única y exclusivamente sobre el estado anterior; es decir: sobre la condición físico-química de la que procede cada fenómeno, determinando inmediatamente su manifestación. Cuando el experimentador enfría un cuerpo líquido para que se cristalice, él no actúa sobre la cristalización, que es una propiedad inherente a la materia mineral, él sólo determina la condición bajo la cual dicha cristalización tiene lugar. Cuando se calienta a 100 grados cloruro de nitrógeno y se produce una explosión, que es a la vez fuente poderosa de movimiento y de calor, no se está actuando sobre la explosión en sí: lo único que se hace es producir una temperatura de 100 grados que es la condición determinante para que se produzca la explosión.

Para los fenómenos orgánicos es exactamente lo mismo. Cuando se ponen, por ejemplo, glóbulos de levadura de cerveza en un líquido azucarado, y se los mantiene a una temperatura inferior a 10 grados, nada ocurre en el líquido: la levadura adormecida no reacciona con el azúcar, y no se forman ni ácido carbónico ni alcohol. Pero, si elevamos la temperatura a más de 30 grados, vemos a la fermentación avanzando con rapidez. En este caso, sin embargo, tampoco hemos actuado sobre la propiedad de la fermentación que es esencial e inherente a la levadura, sino que simplemente produjimos las condiciones químico-físicas en las cuales la fermentación de detiene o se manifiesta. Y si tomásemos ejemplos en los fenómenos más complejos y más misteriosos de los seres vivos, veríamos que la aplicación de la experimentación debe entenderse de la misma manera.

Lo que pasa cada día bajo nuestros ojos durante la incubación de un huevo de gallina debería servir para maravillarnos y para mostrarnos la profundidad de nuestra ignorancia. Pero no nos sorprendemos ante los fenómenos comunes porque no reflexionamos sobre ellos. Se ha comparado a la evolución orgánica silenciosa que tiene lugar en un huevo con la armonía de un cuerpo celeste en el espacio. Van Helmont, apareciendo como un espíritu lúcido en el medio de las tinieblas de la Edad Media, había ubicado en el huevo un archeus faber, o una idea, que dirigía la evolución. ${ }^{10}$ Eso se parece, en efecto, a una idea que se desarrolla, ya que desde ese momento todo está coordinado, todo está previsto, no sólo para la evolución del nuevo ser, sino también para su manutención funcional durante toda su vida, porque la nutrición es la generación continua.

Y si recurriésemos a la ciencia moderna, veríamos que, en el huevo, la parte esencial se reduce a una pequeña vesícula o célula microscópica. El resto del huevo del pájaro, la yema y la clara, no son más que materiales nutritivos destinados a proveer al desarrollo que debe tener lugar fuera del cuerpo maternal. Estamos entonces obligados a postular que, dentro de la simple célula orgánica microscópica que compone al huevo de todos los animales, hay una idea evolutiva tan compleja que no sólo encierra todos los caracteres específicos del ser, sino que además traza todos los detalles del individuo. Es así que una enfermedad que aparecerá en el hombre por herencia veinte o treinta años más tarde, se encuentra ya en germen en esta vesícula misteriosa. Pero esta idea específica contenida en el huevo no se manifiesta y no se desarrolla por sí misma. Ella lo hace bajo la influencia de ciertas condiciones puramente físico-químicas. Como nuestra célula de levadura de cerveza, la célula del huevo se adormece bajo cierta temperatura y únicamente por encima de los 35 grados la idea orgánica manifestará su actividad.

Me detengo aquí. Los ejemplos ya citados, que se refieren todos a hechos bien conocidos, me parecen suficientes para expresar mi sentimiento y dar a entender mi pensamiento. El experimentador, o el determinista, debe observar los fenómenos de la naturaleza sólo para encontrar su causa determinante; sin querer explicarlos por sus causas primeras recurriendo a sistemas que pueden llenarlo de orgullo pero que no hacen más que disimular su ignorancia. Hay que cesar de establecer una diferencia entre los fenómenos

\footnotetext{
${ }^{10}$ Ver J. Guislain, tesis sobre Van Helmont, la Naturaleza, etc., p. 164.
} 
de los cuerpos vivientes y los fenómenos de los cuerpos brutos que se pretenda fundada en el supuesto hecho de que podemos conocer la naturaleza de los primeros, ignorando la de los segundos. La verdad es que la naturaleza o la esencia de todos los fenómenos, vitales o minerales, nos es siempre completamente desconocida. La esencia del fenómeno mineral más simple es ignorada por el químico y el físico, de la misma manera que el fisiólogo ignora la esencia de los fenómenos intelectuales o la causa primera de un fenómeno vital cualquiera.

Sabemos, además, que el conocimiento de la naturaleza íntima de las cosas, o el conocimiento de lo absoluto, exigiría, aun para el fenómeno más simple, el conocimiento del universo entero; porque es evidente que cualquier fenómeno del universo es siempre un parte de este universo en cuya armonía él está necesariamente integrado. El conocimiento del absoluto sería, entontes, el conocimiento que no dejaría nada fuera; y, aunque el hombre desee obtenerlo, está claro que no podrá poseerlo mientras ignore algo. Y la razón parece decirnos que será siempre así. De todas maneras, aun corrigiéndolo, la razón no hace desaparecer ese sentimiento. El hombre, aun corrigiéndose, no cambia su naturaleza, su sentimiento reprimido reaparece y se muestra en otra parte. Es por eso que la experiencia, que constantemente le muestra al científico que su conocimiento está limitado, no llega nunca a sofocar el sentimiento natural que hace que él crea que la verdad absoluta está dentro de su dominio. El hombre se comporta instintivamente como si debiera lograrlo; y el porqué, que incesantemente le plantea a la naturaleza, así lo prueba.

Por otra parte, sería malo para la ciencia que la razón o la experiencia sofocasen completamente el sentimiento o el anhelo de lo absoluto. El científico iría más allá del objetivo del método experimental, haciendo como aquel que, para enderezar una rama y corregir su dirección, la rompiera, deteniendo así la savia y el crecimiento. Conforme lo veremos después, es esa esperanza de la verdad, siempre frustrada pero siempre renaciente, lo que sostiene y sostendrá a las generaciones sucesivas en su ardor apasionado por estudiar los fenómenos de la naturaleza.

El rol particular que juega la ciencia experimental es enseñarnos que ignoramos, mostrándonos claramente que el límite de nuestros conocimientos es el determinismo. Pero, como una compensación maravillosa, a medida que la ciencia contraría nuestros sentimientos y rebaja nuestro orgullo, ella también aumenta nuestro poder. El científico que llevó el análisis experimental hasta el determinismo de un fenómeno, ve claramente que él ignora la causa primera de dicho fenómeno, pero a aun así lo puede manejar. El instrumento que utiliza permanece desconocido en su esencia, pero él sabe cómo utilizarlo. Ignoramos la esencia del fuego, de la electricidad, de la luz, pero no obstante eso, controlamos esos fenómenos para nuestro provecho. Ignoramos la esencia de la vida, pero controlamos los fenómenos vitales a partir del momento en que conocemos suficientemente sus condiciones de existencia. La única diferencia es que en los fenómenos vitales el determinismo es mucho más difícil de alcanzar porque las condiciones son infinitamente más complejas y delicadas, y están combinadas unas con otras.

El físico y el químico que no se ubican fuera del universo, pueden estudiar los cuerpos y los fenómenos aisladamente para entenderlos. Ellos no están obligados a relacionar esos fenómenos con el conjunto de la naturaleza. Pero el fisiólogo, por el contrario, que sí se encuentra fuera del organismo animal del cual sólo puede ver el conjunto. Él debe tomar en cuenta la armonía de este conjunto; y, al mismo tiempo, también debe tratar de penetrar en su interior para analizar el mecanismo de cada una de sus partes. Por eso, mientras que el químico y el físico pueden rechazar toda idea de causas finales en los hechos que ellos observan, el fisiólogo, por el contrario, tiene que admitir una finalidad armónica y preestablecida en el cuerpo organizado, cuyas acciones parciales son solidarias y generadoras las unas de las otras. Si con la ayuda del análisis experimental, se descompone el organismo vivo, aislando sus diversas partes, no es para concebirlas por separado. Cuando se le quiere dar a la propiedad fisiológica de un órgano, o de un tejido, todo su valor 
y su verdadero significado, hay que relacionarlo siempre con el organismo, sin sacar conclusiones que no refieran a sus efectos dentro del conjunto organizado. Hay que reconocer que el determinismo en los fenómenos de la vida no sólo es muy complejo, sino que al mismo tiempo es un determinismo armónicamente subordinado. Los fenómenos fisiológicos, que son tan complicados en los animales superiores, están constituidos por una serie de fenómenos simples que se engendran unos a otros, asociándose y dándose continuidad en virtud de un objetivo final común.

El objetivo esencial del fisiólogo es determinar, por medio del análisis experimental, las condiciones elementales de los fenómenos fisiológicos complejos y también entender su subordinación natural con el fin de comprender y poder seguir las diversas combinaciones de los variados mecanismos que se dan en los seres vivos. El antiguo emblema, representado por una serpiente que forma un círculo mordiéndose la cola, nos da una imagen bastante acertada de la vida. Efectivamente, el organismo vital forma un circuito cerrado, pero este círculo tiene una cabeza y una cola; y, en ese sentido, puede decirse que no todos los fenómenos vitales tienen la misma importancia, aun cuando ellos estén todos conectados y contribuyan al circulus vital. Los órganos musculares y nerviosos mantienen en actividad a los órganos que preparan la sangre o el medio interior; pero la sangre, por su parte, nutre a los órganos que la producen. Hay una solidaridad orgánica y social que constantemente mantiene, en la economía animal, un movimiento de desgaste y renovación que perdura hasta el momento en que la alteración, o el cese de la acción, de un elemento orgánico necesario, provoque un trastorno en el funcionamiento de la maquinaria viva o resulte en su detención definitiva.

El problema del médico experimentador consiste pues, en encontrar el determinismo simple de un desarreglo orgánico complicado; es decir: él debe descubrir la condición de ese fenómeno patológico inicial del que resultan todos los otros por la mediación de un determinismo complejo que no es otra cosa que una suma de determinismos simples. El determinismo del fenómeno inicial, una vez encontrado, será el hilo de Ariadna que guiará al experimentador, permitiéndole saber en qué lugar dentro del laberinto, aparentemente tan oscuro de los fenómenos fisiológicos y patológicos, él de hecho se encuentra. Él comprenderá así de qué manera, una sucesión de determinismos subordinados unos a otros, puede engendrar un conjunto lógico de fenómenos que se reproducen siempre de la misma forma, como si fuesen individualidades que pertenecen a una misma especie definida. En estado fisiológico estos tipos de fenómenos constituyen las funciones, y, en estado patológico, ellos conforman las enfermedades. El origen de una enfermedad, según Van Helmont, se debía a la evolución de una idea mórbida (idea febrilis), y para los médicos de hoy sigue siendo la expresión de una entidad mórbida. Sin embargo, tanto los envenenamientos, como las enfermedades, remiten a un determinismo complejo cuyo determinismo inicial es la acción físico-química del veneno sobre un elemento organizado; aunque, a continuación, en los determinismos secundarios puedan intervenir condiciones de fenómenos que podemos llamar vitales porque ellas no se producen fuera del organismo vivo sano o enfermo. ${ }^{11}$

El conocimiento del determinismo físico-químico inicial de los fenómenos complejos fisiológicos o patológicos, permitirá que el fisiólogo actúe racionalmente sobre los fenómenos de la vida, extendiendo sobre ellos su poder tal como lo hacen el físico y el químico sobre los fenómenos de los cuerpos brutos. Pero no podemos abusar de nuestro poder; pues, en vez de mandar sobre la naturaleza, nosotros le obedecemos. No podemos conocer los fenómenos de la naturaleza si no es por su relación con su causa determinante o próxima; y la ley no es otra cosa que esta relación establecida numéricamente que permite prever la relación de la causa con el efecto en todos los casos dados. Esta relación establecida por la observación, que permite

\footnotetext{
${ }^{11}$ Podría citar muchos ejemplos para probar esto que afirmo. Me limitaré a recordar mis investigaciones sobre la acción del curare en las cuales se puede ver como la lesión física extremidad nerviosa motriz afecta sucesivamente a todos los otros elementos vitales y provoca determinismos secundarios que se van complicando progresistamente hasta provocar la muerte.
} 
al astrónomo predecir los fenómenos celestes, es la misma relación, establecida por la observación y el experimento, que permite al físico, al químico y al fisiólogo, no solamente predecir los fenómenos de la naturaleza, sino también modificarlos a voluntad, pero siempre respetando las relaciones que la experiencia indicó, es decir, la ley. Esto quiere decir, en otros términos, que no podemos gobernar a los fenómenos de la naturaleza si no es sometiéndonos a las leyes que los rigen.

El experimentador no puede cambiar las leyes de la naturaleza. El actúa sobre los fenómenos cuando conoce su determinismo físico-químico, pero no puede ni crear ni aniquilar esos fenómenos: sólo puede modificarlos. Las condiciones físico-químicas de los fenómenos son más fáciles de analizar y de precisar cuando los fenómenos son más simples. Pero como ya fue dicho, la causa primera del fenómeno sigue siendo, en el fondo y en todos los casos, totalmente impenetrable. El experimentador puede más de lo que realmente sabe, y cualquiera sea la manera en que su espíritu concibe las fuerzas de la naturaleza, vitales o minerales, su problema es siempre el mismo: determinar las condiciones materiales en la cuales el fenómeno aparece; para, una vez conocidas esas condiciones, actuar o no, haciendo aparecer o desaparecer el fenómeno. Para producir un fenómeno nuevo, el experimentador recrea las condiciones del fenómeno, pero él no crea nada: ni fuerza, ni materia.

A finales del siglo pasado la ciencia proclamó una gran verdad: en lo que concierne a la materia, en la naturaleza nada se pierde, ni nada se crea. Todos los cuerpos cuyas propiedades varían sin cesar bajo nuestros ojos, no son más que transmutaciones de mezclas de materias equivalentes en peso. Ya en los últimos tiempos, la ciencia proclamó una segunda verdad cuya demostración todavía sigue en proceso y que es, en cierta forma, complementaria de la primera: en cuanto a las fuerzas, nada se pierde y nada se crea en la naturaleza. Deduciéndose de ahí que todas las formas de los fenómenos del universo, variadas hasta el infinito, no son más que trasformaciones de fuerzas equivalentes las unas en las otras. Sin querer abordar aquí una cuestión de la naturaleza de las fuerzas minerales y de las fuerzas vitales, me basta decir que las dos verdades que acabo de anunciar son universales y que ellas abarcan los fenómenos de los cuerpos vivos tanto como los de los cuerpos brutos.

Como consecuencia de lo que precede, vemos que todos los fenómenos, de cualquier orden que sean, existen virtualmente, en las leyes inmutables de la naturaleza y sólo se manifiestan cuando sus condiciones de existencia se realizan. Los cuerpos y los seres que están en la superficie de nuestra Tierra expresan la relación armoniosa de las condiciones cósmicas de nuestro planeta y de nuestra atmósfera con los seres y los fenómenos cuya existencia dichas condiciones permiten. Otras condiciones cósmicas harían, necesariamente, aparecer otro mundo en el cual se manifestarían todos los fenómenos que encontrarían allí sus condiciones de existencia, y del cual desaparecerían los que no pudieran desarrollarse. Pero sean como sean las variedades infinitas de fenómenos que concebiríamos como pasibles de darse en la Tierra, ubicándonos con el pensamiento en todas las condiciones cósmicas que nuestra imaginación pueda crear, siempre estaríamos obligados a admitir que ahí todo ocurría según las leyes de la física, de la química y de la fisiología ahora existentes, y también que deberíamos admitir que allí no habría nada creado en lo que respecta a la fuerza y la materia. Ahí sólo habría producción de relaciones diferentes, y en consecuencia creación de seres y de fenómenos nuevos.

Cuando un químico hace aparecer un cuerpo nuevo en la naturaleza, él no puede jactarse de haber creado las leyes que han hecho nacer ese cuerpo. Él simplemente recreó las condiciones que eran exigidas por la ley creadora para manifestarse; y lo mismo sucede con los cuerpos organizados: un químico y un fisiólogo sólo podrían hacer aparecer seres vivos con sus experimentos obedeciendo a las leyes eternas de la naturaleza. 
El método experimental tiene como objetivo encontrar el determinismo o la causa próxima de los fenómenos de la naturaleza. El principio sobre el cual se basa este método es la certeza de que un determinismo existe, su procedimiento de investigación es la duda filosófica, y su criterio es la experiencia. O dicho en otros términos: el científico cree de manera absoluta en la existencia del determinismo que él investiga; pero siempre duda de haberlo encontrado. Es por eso que él se ve constantemente obligado a acudir a la experiencia. El método experimental no es más que la expresión de la marcha natural del espíritu humano en la investigación de las verdades científicas que están fuera de nosotros. Cada hombre se hace una idea de lo que ve y tiende a interpretar los fenómenos de la naturaleza antes de conocerlos por la experiencia. Esta tendencia es espontánea: una idea preconcebida siempre fue y será el primer impulso de un espíritu investigador. El método experimental tiene por objeto transformar este concepto a priori, fundado sobre la intuición o sobre un sentimiento vago de las cosas, en una interpretación a posteriori, establecida sobre el estudio experimental de los fenómenos. Es por eso que al método experimental también se lo llama método a posteriori.

El espíritu humano ha pasado por tres períodos necesarios en su evolución. Primero el sentimiento que, imponiéndose a la razón, creó las verdades de la fe; es decir: la teología. Después la razón, o la filosofía, fue el ama; creando los sistemas o la escolástica. Finalmente, la experiencia, es decir, el estudio de los fenómenos naturales, le enseñó al hombre que las verdades del mundo exterior no están fundadas ni el sentimiento, ni en la razón, que sólo son nuestras guías indispensables; y también le enseñó que, para alcanzar esas verdades, es necesario descender a la realidad objetiva de los hechos en donde éstos se encuentran bajo la forma de relaciones fenoménicas. Fue así, por el progreso natural de las cosas, que apareció el método experimental; que resume todo apoyándose sucesivamente sobre las tres ramas de este trípode inmutable: el sentimiento, la razón y la experiencia. En la búsqueda de la verdad por medio de este método, el sentimiento tiene siempre la iniciativa, pues él engendra la idea a priori: es la intuición. La razón o el razonamiento desarrolla, a continuación, la idea y deduce sus consecuencias lógicas. Pero, si el sentimiento debe ser aclarado por las luces de la razón; y ésta, a su vez, debe ser guiada por la experiencia que es la única que le permite llegar a una conclusión. El espíritu humano es un todo complejo que sólo marcha y funciona por el juego armónico de sus diversas facultades.

En la asociación que señalé antes, hay que tener cuidado de no dar una predominancia exagerada ni al sentimiento, ni a la razón, ni tampoco a la experiencia. Si el sentimiento acalla la razón, estamos fuera de la ciencia, y así llegamos a verdades irracionales de la fe o de la tradición. Si la razón no invoca constantemente a la experiencia, caemos en la escolástica y bajo la dominación de los sistemas; y si la experiencia deja de lado el razonamiento, no podemos salir de los hechos, estancándonos en el empirismo. El método experimental es el método que busca la verdad con el empleo bien equilibrado del sentimiento, la razón y la experiencia. Él proclama la libertad de espíritu y de pensamiento. Su característica saliente es la de sólo someterse a sí mismo, atribuyendo a su criterio, que es la experiencia, una autoridad impersonal que domina a toda la ciencia. El método experimental no admite autoridades personales, y rechaza de manera absoluta los sistemas y las doctrinas. Pero eso no es orgullo ni jactancia. El experimentador, por el contrario, hace un acto de humildad negando la autoridad individual, porque él duda de sus propios conocimientos, y somete la autoridad de los hombres a la autoridad de la experiencia y a la autoridad de las leyes de la naturaleza.

La primera condición que debe cumplir un científico que se dedica a la investigación experimental de los fenómenos naturales es no preocuparse por ningún sistema, conservando una total libertad de espíritu basada en la duda filosófica. En efecto, por un lado, tenemos la certeza de la existencia del determinismo de 
los fenómenos, pues esta certeza nos está dada por la relación necesaria de causalidad de la que nuestro espíritu es consciente; pero, por otra parte, no tenemos ninguna certeza relacionada con la fórmula de este determinismo porque él se realiza en fenómenos que son ajenos a nosotros. Sólo la experiencia debe guiarnos, ella es nuestro único criterio y, conforme a la expresión de Goethe, ${ }^{12}$ ella es la única mediadora que existe entre el científico y los fenómenos que lo rodean. Una vez que la investigación del determinismo de los fenómenos es admitida como único objetivo del método experimental, no hay más materialismo, ni espiritualismo, ni materia bruta, ni materia viva: sólo hay fenómenos naturales de los que hay que determinar las condiciones. Es decir: fenómenos de los que hay conocer esas circunstancias que constituyen sus causas próximas. Todas las ciencias que utilizan el método experimental deben tender a devenir anti-sistemáticas; y, por eso, la medicina experimental no será un nuevo sistema de medicina, sino que será la negación de todos los sistemas. Ella no deberá aferrarse a ninguna palabra sistemática: no será ni animista, ni organicista, ni solidista, ni humoral; ella será simplemente la ciencia que trata de analizar las causas próximas de los fenómenos tanto en el estado sano y como en el estado mórbido.

Y esto que acabamos de decir respecto a los sistemas médicos, lo podemos aplicar a los sistemas filosóficos. La fisiología experimental no tiene necesidad de unirse a ningún sistema filosófico. El rol del fisiólogo, como el de todo científico, es buscar la verdad por la verdad misma; sin querer ponerla al servicio de ningún sistema de filosofía. Cuando el científico conduce su investigación partiendo de la base de un sistema filosófico cualquiera, él siempre se pierde en las regiones de las causas primeras. La idea sistemática le da al espíritu una especie de seguridad engañosa y una inflexibilidad que no concuerdan con la libertad de la duda que siempre debe conservar el experimentador en sus investigaciones. Los sistemas son siempre necesariamente incompletos: no pueden representar todo lo que hay en la naturaleza, sino solamente lo que ya está en el espíritu de los hombres. Para encontrar la verdad basta con que el científico enfrente la naturaleza, interrogándola libremente siguiendo el método experimental y valiéndose de recursos de investigación cada vez más perfectos. En este caso, pienso, el único sistema filosófico consiste en no tener ninguno.

Como experimentador, yo evito los sistemas filosóficos, pero no por eso sería capaz de rechazar este espiritu filosófico que, sin estar en ningún lugar está en todas partes y que sin pertenecer a ningún sistema debe reinar, no solamente sobre las ciencias, sino sobre todos los conocimientos humanos. Es eso lo que hace que, aun huyendo de los sistemas filosóficos, yo ame mucho a los filósofos, disfrutando infinitamente de su trato. Efectivamente, desde el punto de vista científico, la filosofía representa la aspiración eterna de la razón humana hacia el conocimiento de lo desconocido. Por eso los filósofos se ocupan siempre de las cuestiones controversiales que se plantean en esas regiones más elevadas que son los límites superiores de las ciencias. Los filósofos comunican al pensamiento científico un movimiento que lo reaviva y lo ennoblece. Ellos fortalecen al espíritu desarrollándolo por medio de una gimnasia intelectual general, y al mismo tiempo lo empujan sin cesar hacia las soluciones inagotables de los grandes problemas. Eso mantienen una especie de sed de lo desconocido, y ese fuego sagrado de la investigación, que nunca debe apagarse en un científico.

En efecto, el deseo ardiente del conocimiento es el único móvil que atrae y mantiene al investigador en sus esfuerzos; y es precisamente este conocimiento, que él atrapa y que siempre huye siempre delante de él, lo que constituye, al mismo tiempo, su único tormento y su única felicidad. El que no conoce los tormentos de lo desconocido, ignora esas alegrías del descubrimiento que son, sin duda, las más fuertes que el espíritu del hombre puede sentir. Aunque, por un capricho de nuestra naturaleza, esta alegría del descubrimiento tan buscado y tan esperado se desvanece cuando se lo logra. Es sólo un relámpago cuya luz nos descubre

\footnotetext{
${ }^{12}$ Goethe, Obras de historia natural, traducción de M. Martins, introducción, p. 1.
} 
otros horizontes hacia los cuales nuestra curiosidad insaciable se dirige con más ardor aún. Es eso lo que hace que, en la ciencia, lo conocido pierda su atractivo mientras que lo desconocido permanece lleno de encantos. Es por eso que los espíritus que se elevan y llegan a ser verdaderamente grandes son los que nunca están satisfechos con las obras por ellos realizadas, procurando siempre a mejorarlas con obras nuevas.

El sentimiento al que me refiero en este momento, es bien conocido por científicos y filósofos. Es este el sentimiento que le hizo decir a Priestley ${ }^{13}$ que un descubrimiento que hacemos nos muestra muchos otros por hacer; y es el sentimiento que Pascal ${ }^{14}$ expresa como una paradoja, cuando dice: "nosotros nunca buscamos las cosas, investigamos las cosas”. Sin embargo, es la verdad la que nos interesa; y, si siempre estamos en su búsqueda, es porque lo que encontramos no nos termina de satisfacer. Si no fuera así, nuestras investigaciones serían el trabajo inútil e interminable que representa la fábula de Sísifo; que una y otra vez hace rodar una roca cuesta arriba hasta la cima de una montaña, pero, una vez que llaga ahí, la roca siempre termina rodando cuesta abajo, volviendo a su punto de partida. Esta comparación no es científicamente exacta: el científico asciende siempre buscando la verdad. Y si bien nunca la encuentra toda entera, aun así él descubre fragmentos de ella que son muy importantes; siendo precisamente esos pedazos de la verdad general, lo que constituye la ciencia.

El científico no investiga por el placer de buscar sino por el placer de encontrar. Busca la verdad por el deseo ardiente de poseerla y la posee dentro de los límites que fijan las ciencias en su estado actual. Pero el científico no debe detenerse en el camino. Él debe tratar de llegar cada vez más alto, tendiendo a la perfección; y, mientras vislumbre algo que merezca ser encontrado, debe continuar investigando. Sin esta excitación constante, provocada por el acicate de lo desconocido, sin esta sed de saber siempre renovada, el científico corre el peligro de sistematizarse en lo ya adquirido y conocido. La ciencia, así, no haría más progresos y se detendría por indiferencia intelectual, como cuando los cuerpos minerales saturados caen en la indiferencia química y se cristalizan.

Hay que impedir que el espíritu, muy absorbido por lo ya conocido de una ciencia especial no tienda a descansar, o se arrastre sin imaginación perdiendo de vista las cuestiones que aún quedan por resolver. La filosofía, agitando la masa inagotable de cuestiones no resueltas, estimula y mantiene este movimiento saludable en las ciencias, porque, en el sentido restringido en el que yo considero aquí a la filosofía, a ella sólo le compete lo indeterminado: lo determinado entra necesariamente en el dominio científico. Yo no admito, por lo tanto, ninguna filosofía que quiera ponerle límites a la ciencia; como tampoco admitiría una ciencia que pretendiese suprimir las verdades filosóficas que, en la actualidad, están fuera de su propio dominio. La verdadera ciencia no suprime nada, trata siempre de mirar de frente y sin turbarse, las cosas que todavía no entiende. Negar estas cosas no sería suprimirlas, seria cerrar los ojos y creer que la luz no existe. Sería la ilusión del avestruz que cree suprimir el peligro escondiendo la cabeza en la arena.

Yo creo que el verdadero espíritu filosófico es aquel cuyas aspiraciones más elevadas fecundan a las ciencias arrastrándolas a la investigación de las verdades que en la actualidad están fuera de ellas, pero que no deben ser abandonadas aun cuando ellas se alejen, o se eleven, conforme van siendo abordadas por espíritus filosóficos más poderosos y más refinados. ¿Tendrá fin esa aspiración del espíritu humano? ¿Encontrará un límite? No puedo saberlo; pero el científico sólo puede seguir caminando, sin cesar, porque así siempre avanza.

Uno de los mayores obstáculos que se encuentra en esta marcha general y libre del conocimiento humano es la tendencia que hace que conocimientos diversos se aíslen en sistemas. Esto no es una consecuencia de la propia naturaleza de las cosas; porque en la naturaleza todo está conectado y nada puede

${ }^{13}$ Priestley, Experimentos y observaciones en diferentes tipos de aire, t. 1, prefacio, p. 15.

${ }^{14}$ Pascal, Pensamientos morales destacados, art. IX-XXXIV. 
ser considerado de forma aislada y sistemática. Pero, como resultado de una tendencia de nuestro espíritu, al mismo tiempo débil y dominador, somos llevados a absorber los otros conocimientos en una sistematización personal. Una ciencia que se detuviera en un sistema sería estacionaria y se aislaría; porque la sistematización es un verdadero enquistamiento científico, y toda parte enquistada de un organismo deja de participar de la vida general de ese organismo.

Los sistemas tienden a esclavizar al espíritu humano; y, según entiendo, la única utilidad que podemos encontrar en ellos es la de suscitar combates que los destruyen agitando y excitando la vitalidad de las ciencias. En efecto, hay que tratar de romper los obstáculos de los sistemas filosóficos y científicos, como romperíamos las cadenas de la esclavitud intelectual. La verdad, si podemos encontrarla, pertenece a todos los sistemas; y para descubrirla el experimentador necesita moverse libremente, por todas partes, sin sentirse impedido por las barreras de un sistema cualquiera. La filosofía y la ciencia no deben ser sistemáticas; ellas deben estar unidas y ayudarse mutuamente sin tratar de dominarse la una a la otra. Pero, si en lugar de contentarse con esta unión fraternal para la búsqueda de la verdad, la filosofía quisiera introducirse en el medio de la ciencia, imponiéndole dogmáticamente métodos y procedimientos de investigación, ese acuerdo no puede existir. Para realizar observaciones, experimentos o descubrimientos científicos, los métodos y los procedimientos filosóficos son demasiado generales y resultan impotentes. Para esos cometidos hay métodos y procedimientos científicos, a menudo muy especiales, que solamente pueden ser conocidos por los experimentadores, científicos y filósofos, que practican una ciencia determinada.

Los conocimientos humanos están tan entrelazados, y son tan solidarios los unos con los otros en su evolución, que es imposible creer que una influencia individual pueda bastar para hacerlos avanzar si las bases de ese progreso no están en la propia ciencia. Es por eso que, aun reconociendo la superioridad de los grandes hombres, yo pienso que la influencia particular o general que ellos tienen sobre las ciencias siempre está necesariamente en función de su época. Y lo mismo pasa con los filósofos. Ellos sólo pueden seguir la marcha del espíritu humano, contribuyendo con su avance, atrayendo a los espíritus hacia esa vía del progreso que muchos tal vez no perciben pero que también son la expresión de su época. Sería iluso, por lo tanto, pretender absorber los descubrimientos particulares de una ciencia para provecho de un método o de un sistema filosófico cualquiera. En una palabra, si los científicos son útiles a los filósofos, y los filósofos a los científicos, entonces, el científico es libre y dueño de sí mismo. Y, en lo que a mí atañe, pienso que los científicos de laboratorio hacen sus descubrimientos, sus teorías y su ciencia, sin los filósofos. Joseph de Maistre dijo que los que más descubrimientos hicieron en las ciencias son los que menos conocieron a Bacon; ${ }^{15}$ mientras que los que lo leyeron y lo meditaron, al igual que el propio Bacon, no tuvieron mucho éxito.

El arte de develar el determinismo de los fenómenos con la ayuda de procedimientos y métodos científicos sólo se aprende en los laboratorios donde el experimentador se enfrenta a los problemas de la naturaleza. Cuando se está frente a fenómenos de los cuales hay que determinar las condiciones de existencia, o las causas próximas, los procedimientos del razonamiento deben variar hasta el infinito, según la naturaleza de los fenómenos más o menos difíciles, o más o menos complejos, a los que ese razonamiento se aplica. Los especialistas de cada ciencia, son los únicos que pueden intervenir en estos temas, porque no solamente los procedimientos son diferentes, sino porque el espíritu del naturalista no es el mismo que el de fisiólogo, y el del químico no es el del físico.

Cuando los filósofos como Bacon, u otros más modernos, quisieron sistematizar los preceptos para la investigación científica, ellos pudieron parecerles seductores a las personas que ven a las ciencias de lejos.

\footnotetext{
${ }^{15}$ Joseph de Maistre, Examen de la filosofía de Bacon, t. 1, p. 81.
} 
pero, en realidad, obras como ésas no son de ninguna utilidad para los científicos formados; y para aquellos que quieren iniciarse en la cultura de las ciencias, esas obras hacen que ellos se pierdan en una falsa simplificación de las cosas. Más aún: esas obras los incomodan, llenándoles el espíritu de una cantidad de reglas vagas o inaplicables que, si se quiere entrar en la ciencia y ser un verdadero experimentador, es mejor olvidar rápidamente.

Yo creo que en la enseñanza científica el rol del maestro es mostrar experimentalmente al alumno el objetivo que el científico se propone, indicándole todos los medios que puede tener a su disposición para alcanzarlo. A continuación, el maestro debe dejar al alumno libre para que se mueva a su manera, según su naturaleza, para alcanzar el objetivo que le mostró, y sólo debe socorrerlo si ve que se pierde. El verdadero método científico es el que contiene al espíritu sin asfixiarlo: el que, en la medida de lo posible, deja al espíritu frente a sí mismo y lo guía respetando sus cualidades más preciosas, que son la originalidad creadora y la espontaneidad científica. Las ciencias, en efecto, avanzan por las ideas nuevas, y por el poder creador u original del pensamiento. Por eso, en la enseñanza de las ciencias, hay que evitar que los conocimientos que deben armar a la inteligencia no la aplasten por su mismo peso, y que las reglas que están destinadas a sostener las partes más débiles del espíritu no atrofien o asfixien a las partes poderosas y fecundas.

No voy a desarrollar otros temas. Me he limitado aquí a proteger a las ciencias fisiológicas, y la medicina experimental, de las exageraciones de la erudición y de la invasión y la dominación de los sistemas; porque, sucumbiendo a ellas, estas ciencias verían desaparecer su fecundidad y perderían esa independencia y esa libertad de espíritu que siempre serán las condiciones esenciales de su progreso. Si bien el genio del hombre tiene en las ciencias, igual que otras actividades, una supremacía que no pierde jamás sus derechos; aun así, en el caso de las ciencias experimentales, el investigador debe aplicar sus ideas a la búsqueda del determinismo científico, interrogando a la naturaleza en un laboratorio con los medios convenientes y necesarios. No podríamos concebir un físico o un químico sin un laboratorio. Para el fisiólogo debe ser lo mismo: es necesario que él analice experimentalmente los fenómenos de la materia viviente, como el físico y el químico analizan los fenómenos de la materia bruta. Resumiendo, el laboratorio es la condición sine qua non del desarrollo de todas las ciencias experimentales.

La evidencia de esta verdad conduce y conducirá, necesariamente, a una reforma universal profunda en la enseñanza científica; porque hoy ya se ha reconocido en todas partes que es en los laboratorios que germinan y crecen todos los descubrimientos de la ciencia pura, difundiéndose después por el mundo con sus aplicaciones útiles. Sólo el laboratorio puede enseñar las dificultades reales de la ciencia a los que lo frecuentan. Les muestra, además, que la ciencia pura siempre fue la fuente de todas las riquezas reales que el hombre obtuvo y de todas las conquistas que sobre los fenómenos de la naturaleza que él alcanzó. Esa es una excelente educación para la juventud porque sólo ella puede hacerle comprender que las brillantes aplicaciones actuales de las ciencias, no son más que la realización de trabajos anteriores; y que los que hoy aprovechan sus beneficios le deben un tributo de reconocimiento a los que los precedieron: a esos que cultivaron penosamente el árbol de la ciencia sin verlo fructificar.

1 de agosto 1865 .

Claude Bernard 